\title{
Programme Ignobilia Oppida Lucanorum
}

Compte-rendu de la Campagne 2013 : fouille et prospections

Olivier de Cazanove et Stéphane Bourdin

\section{(2) OpenEdition \\ 1 Journals}

\section{Édition électronique}

URL : http://journals.openedition.org/cefr/1194

DOI : $10.4000 /$ cefr. 1194

ISSN : 2282-5703

Éditeur

École française de Rome

Référence électronique

Olivier de Cazanove et Stéphane Bourdin, «Programme Ignobilia Oppida Lucanorum », Chronique des activités archéologiques de l'École française de Rome [En ligne], Italie du Sud, mis en ligne le 16 juillet 2015, consulté le 14 novembre 2019. URL : http://journals.openedition.org/cefr/1194; DOI : 10.4000/ cefr.1194

Ce document a été généré automatiquement le 14 novembre 2019.

(c) École française de Rome 


\section{Programme Ignobilia Oppida Lucanorum}

Compte-rendu de la Campagne 2013 : fouille et prospections

Olivier de Cazanove et Stéphane Bourdin

\section{La fouille autour du petit temple de l'acropole de Civita di Tricarico (Olivier de Cazanove)}

1 La fouille dans la parcelle 50 (publique) de Civita di Tricarico, à l'intérieur de l'aire clôturée sur l'acropole, s'est déroulée du 5 au 17 août 2013. Il s'agissait, en ouvrant des sondages autour du temple fouillé au début des années 70 sous la direction de D. Adamesteanu, de comprendre le rapport entre le lieu de culte et le tissu construit environnant, ainsi que de vérifier l'existence d'une possible phase antérieure au temple, une hypothèse que suggérait le parallèle avec la situation dans la « cité basse ». Là, en effet, la fouille 2003 et 2005 du temple P dans la parcelle 58 avait mis en évidence une phase $\mathrm{du} \mathrm{IV}^{\mathrm{e}} \mathrm{s}$. av. J.-C. au-dessous du temple toscan dépourvu de podium datable à la fin du III ${ }^{e}$-début du II ${ }^{e}$ s. av. J.-C. ${ }^{1}$ Dans la perspective de la publication intégrale du temple $\mathrm{P}$, il était souhaitable de vérifier si la situation était la même sur l'acropole, c'est-à-dire si le petit temple à alae du $\mathrm{II}^{\mathrm{e}} \mathrm{s}$. av. J.-C. avait recouvert un édifice antérieur. Divers indices le laissaient penser : le mobilier trouvé dans le remplissage du podium², mais aussi le fait que celui-ci avait retaillé un mur en pierres sèches ${ }^{3}$.

2 La fouille de 1972 s'était attachée à mettre au jour le petit temple sous l'accumulation de terre, pierraille et fragments divers - entre autres des tuiles portant le timbre Ve.Kar - qui le recouvrait. Il ne s'est pas agi par contre d'une fouille stratigraphique en profondeur (à part un sondage à l'intérieur du podium, contre le mur du fond), si l'on en juge d'après une photographie aérienne zénithale de 1974, conservée à l'IGM de Florence (fig. 1). 
Fig. 1 - Le temple de l'acropole de Civita di Tricarico, photographie aérienne zénithale de 1974.

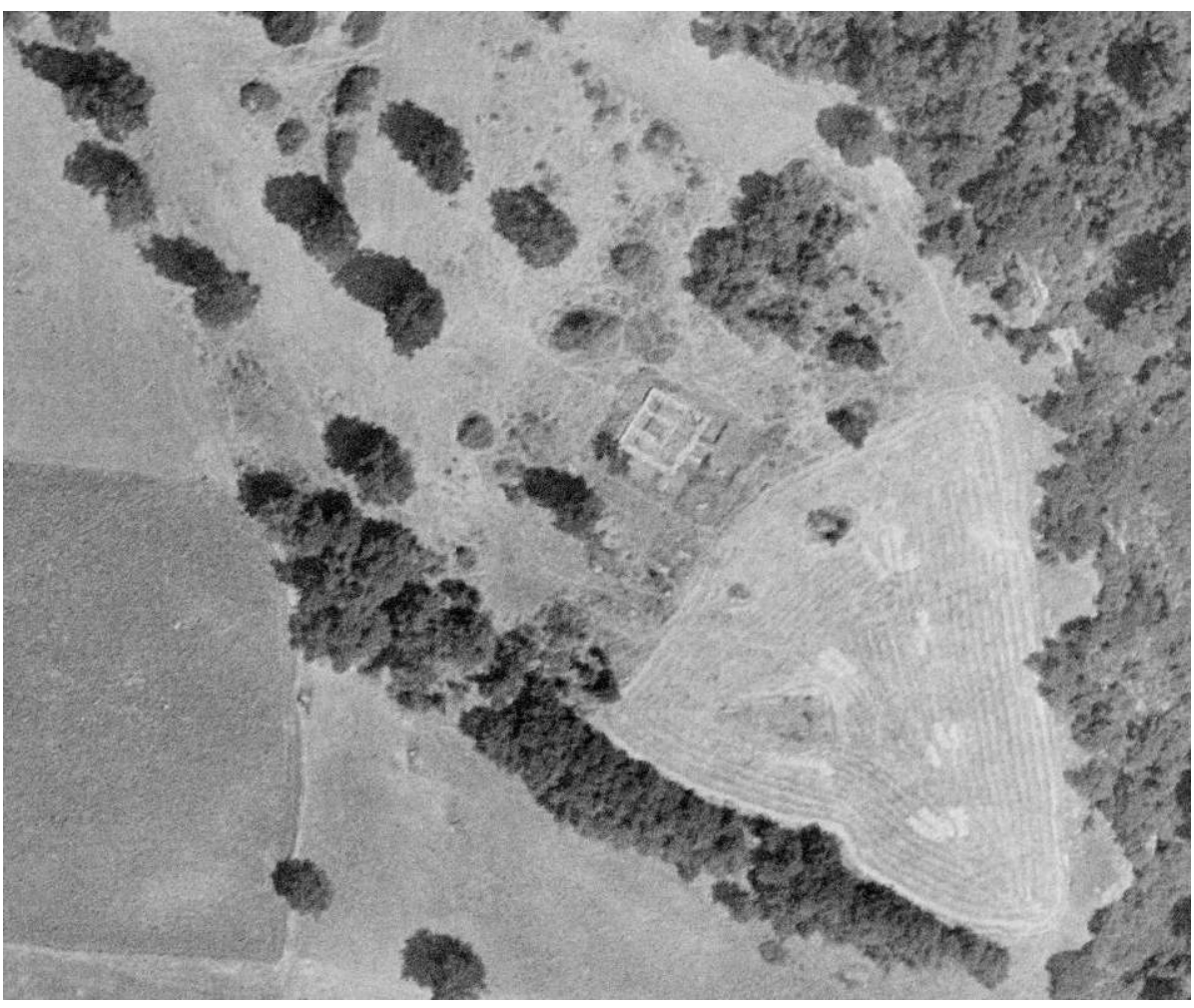

$3338-$ IGM

Dans le décapage effectué autour du temple on entrevoit toutefois, à peu de distance de l'angle sud-ouest du podium, un tronçon de mur en pierres sèches, qui figure sur le relevé réalisé des années après, à l'occasion de la fouille de la domus voisine (en 1985-1986) (fig. 2). 
Fig. 2 - Le temple, plan.

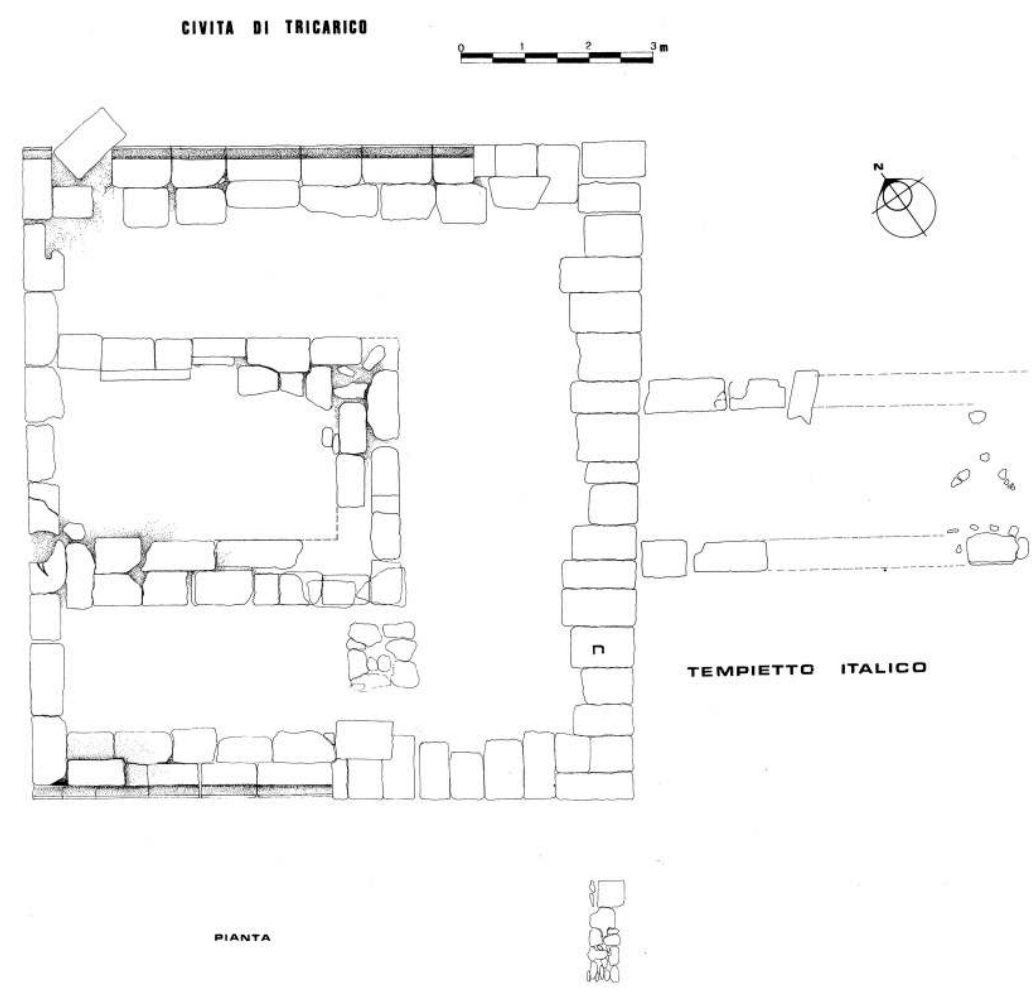

D’après Canosa 1990 ; relevé A. M. Carbone.

4 En 1996, à l'occasion de la reprise des fouilles sur l'acropole (sondage P) un nouveau plan du petit temple a été dressé (fig. 3). 
Fig. 3 - Le temple, plan.

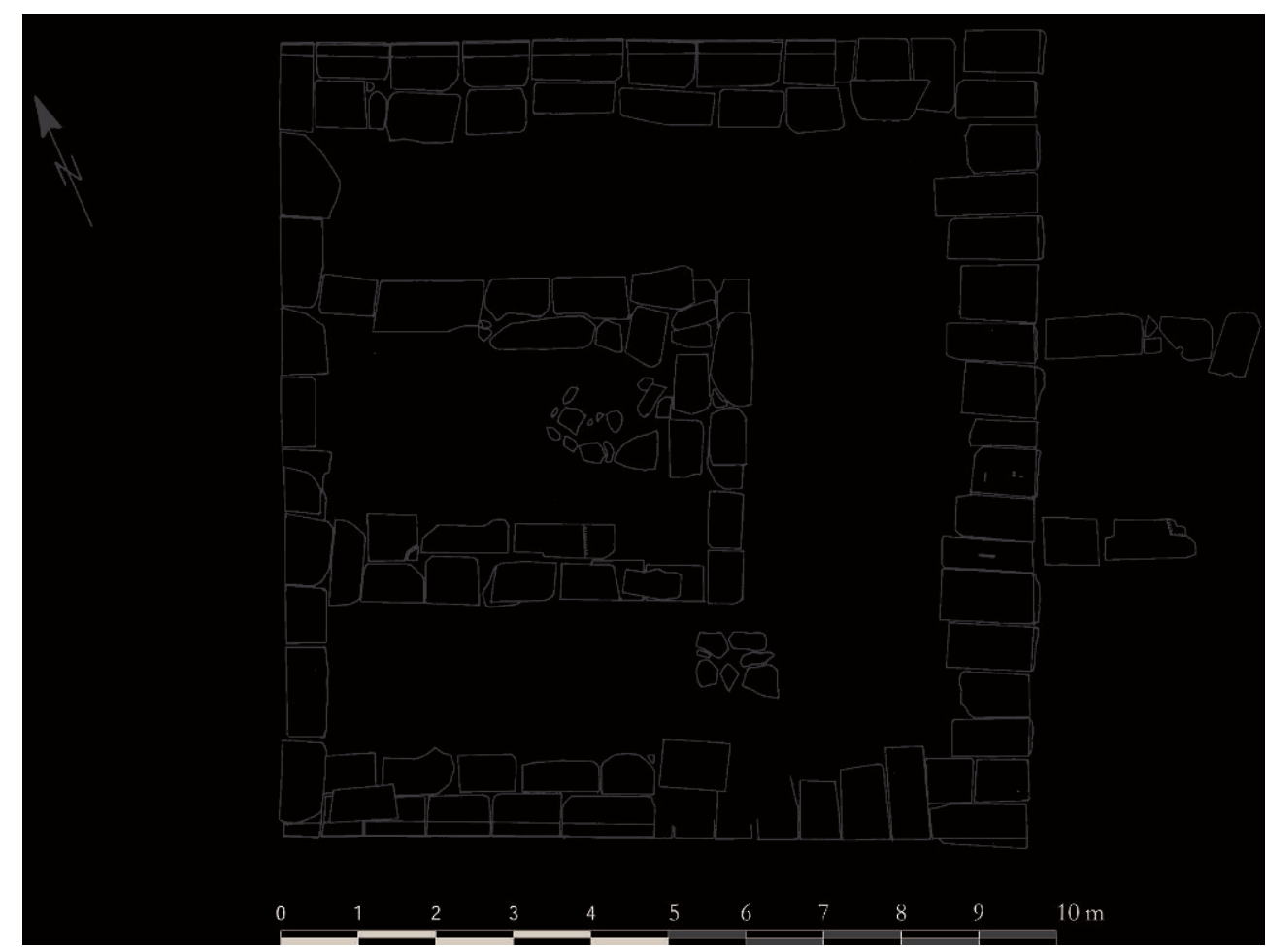

J.-B. Rollin, EFR, 1996.

5 En 2010, une prospection géophysique (électrique et magnétique) conduite sur l'acropole a révélé des traces évanescentes de structures, dont deux alignements derrière le temple (fig. 4). 
Fig. 4 - L'acropole de Civita di Tricarico : structures fouillées et traces révélées par la prospection géophysique de 2010.

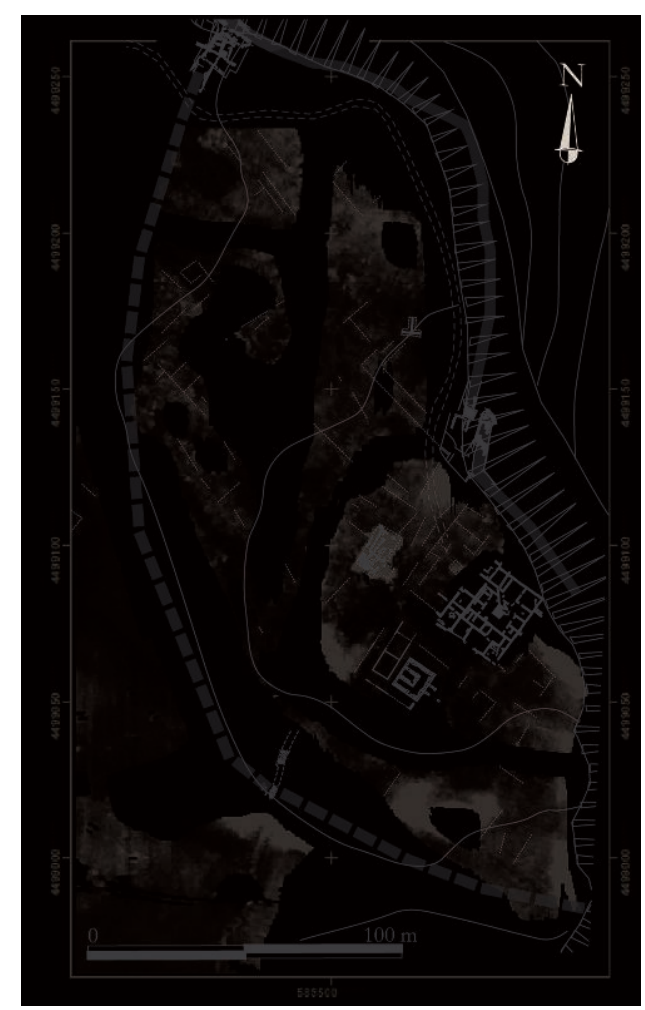

DAO O. de Cazanove.

6 La fouille de 2013 se fixait donc comme objectif prioritaire de vérifier s'il existait ou non une première phase, antérieure au «tempietto ». De fait, les sondages ouverts ont permis de constater que le podium ne s'élevait pas sur un sol vierge, mais qu'il reposait sur et dans une vaste construction arasée, fondée à une cote inférieure. Les murs sont en moellons bruts de calcaire (et parfois de grès), liés avec de l'argile. La technique de construction renvoie aux $\mathrm{IV}^{\mathrm{e}}-\mathrm{III}{ }^{\mathrm{e}} \mathrm{s}$. av. J.-C. Le matériel, peu nombreux et peu significatif, ne permet pas encore une datation plus précise qui devra être obtenue par des sondages stratigraphiques en profondeur.

7 On s'est d'abord attaché à remettre au jour le tronçon de mur déjà connu, qui se trouvait bien à la place attendue. Il s'interrompt à $1,30 \mathrm{~m}$ du podium. Après $2 \mathrm{~m}$ vers le sud-ouest, il plie à angle droit. En même temps on a ouvert une tranchée (3 x 15,5 m), perpendiculaire au mur du fond du podium, de manière à intercepter les anomalies linéaires qui apparaissaient sur la carte de résistivité.

8 Pour le moment, deux côtés d'un grand édifice ont été mis au jour (fig. 5-6 et 10). 
Fig. 5 - Plan de la fouille 2013.

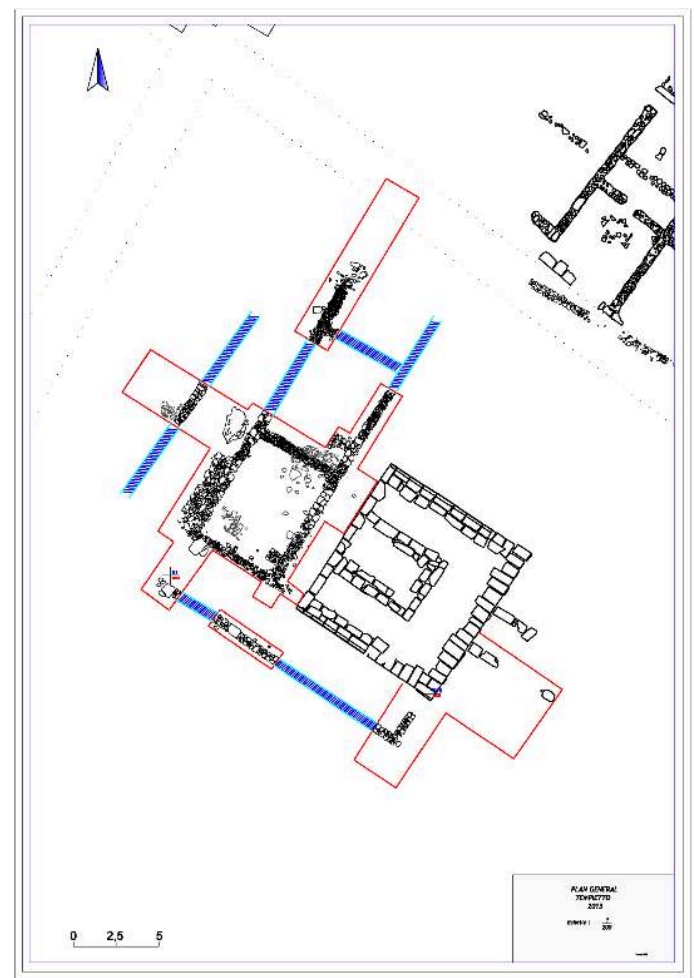

Relevé J. Leblanc, T. Terrasse.

Fig. 6 - Mosaïque orthophotographique du secteur central de l'acropole : le sondage $P$, le temple et la domus avec les sondages 2013.

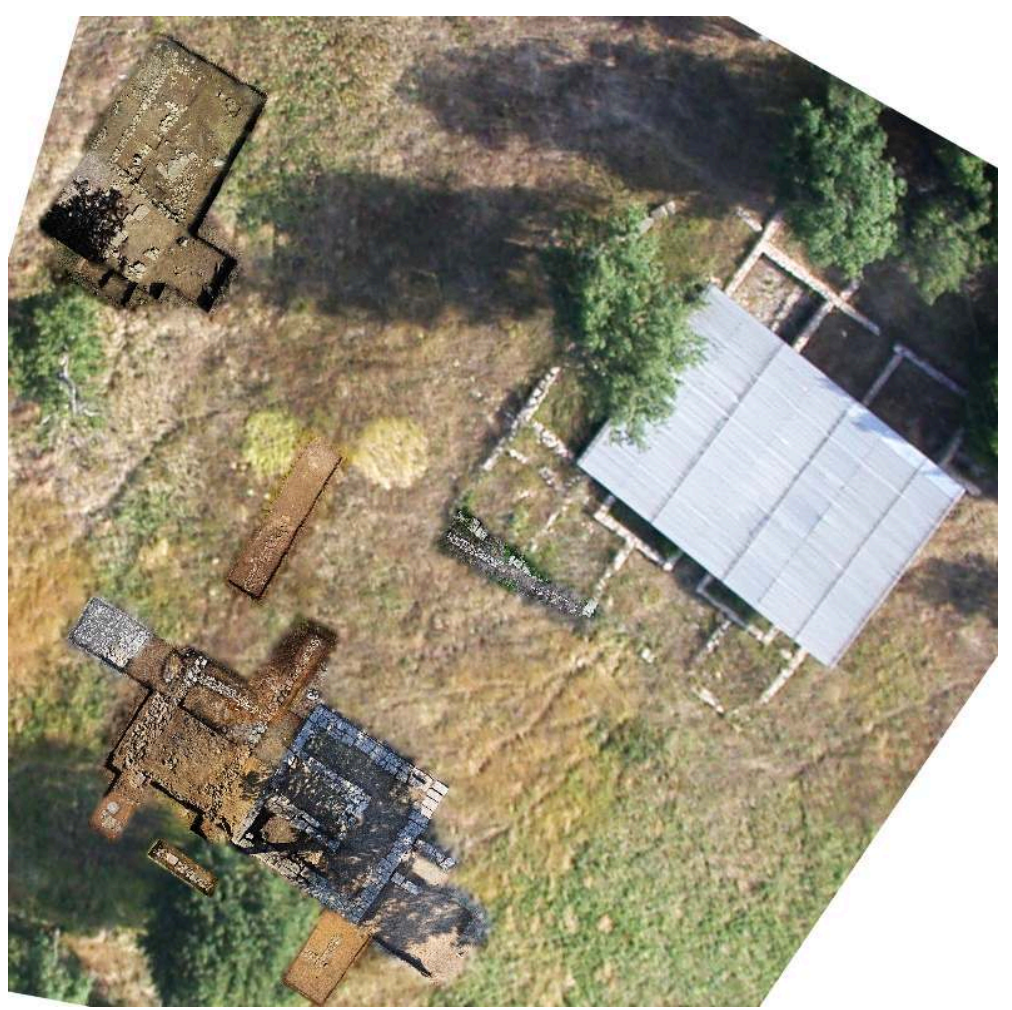

T. Terrasse. 
Le côté sud mesure env. $18 \mathrm{~m}$; on le suit dans trois sondages différents. Il n'est pas lié au mur ouest parce que l'angle est occupé par une baie flanquée de deux bases calcaires. Le côté ouest comptait au moins trois pièces. Les dimensions de deux d'entre elles sont connues et égales entre elles (env. 6,80 x $5 \mathrm{~m}$ ). Plus au nord, l'enquête est rendue difficile par l'état d'épierrement des murs - voire la disparition complète des structures. Pour cette raison, il n'est pas encore possible de dire si un long mur, que la façade de la domus a partiellement recouvert, constituait le côté nord du complexe. Si c'était le cas, on aurait affaire à une vaste construction d'à peu près $18 \times 28 \mathrm{~m}$, soit plus de $500 \mathrm{~m}^{2}$ qui serait, d'après ses dimensions, un édifice communautaire plutôt que domestique. Mais il faut attendre de nouvelles vérifications avant de se prononcer.

On signalera toutefois - puisqu'on pense désormais qu'ils appartiennent à la sphère publique - que la campagne 2013 a livré quatre nouveaux timbres sur tuiles avec l'abréviation Ve.Kar, identiques aux quinze exemplaires déjà connus de l'acropole et de la fouille du « tempietto » (fig. 7).

Fig. 7 - Timbres sur tuiles découverts en 2013.

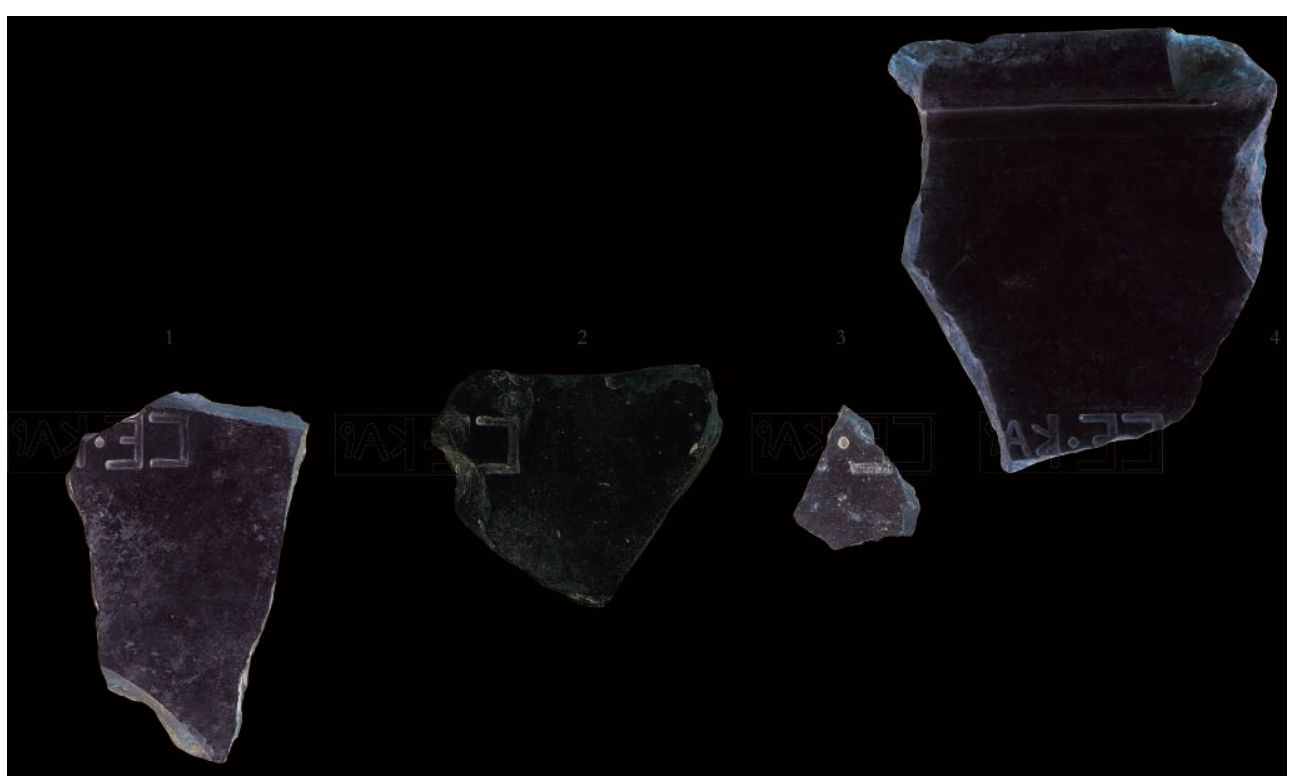

O. de Cazanove.

11 Les quatre nouveaux timbres ont été trouvés dans des strates superficielles, ou bien à l'interface entre celles-ci et les niveaux archéologiques in situ, si bien qu'on ne peut pas encore dire s'ils appartiennent à la première ou à la deuxième phase.

La fouille a également permis de récolter des données importantes sur le temple luimême. Si un sondage ouvert juste devant lui $(6 \times 5 \mathrm{~m})$, sur la moitié de la longueur de sa façade, a atteint l'argile vierge tout de suite sous le niveau humique, sans intercepter ni strates archéologiques ni, moins encore, traces d'aménagements cultuels (un autel ou son négatif auraient été logiquement attendus en ce point), l'abaissement du niveau a $\mathrm{du}$ moins entièrement mis au jour, jusqu'à son plan de pose, le mur d'échiffre sud de l'escalier d'accès au podium. On a pu ainsi constater que l'un de ses blocs était de remploi (fig. 8). Il présentait sur l'un de ses petits côtés une doucine. 
Fig. 8 - Le mur d'échiffre sud de l'escalier d'accès au temple : le bloc mouluré en remploi.

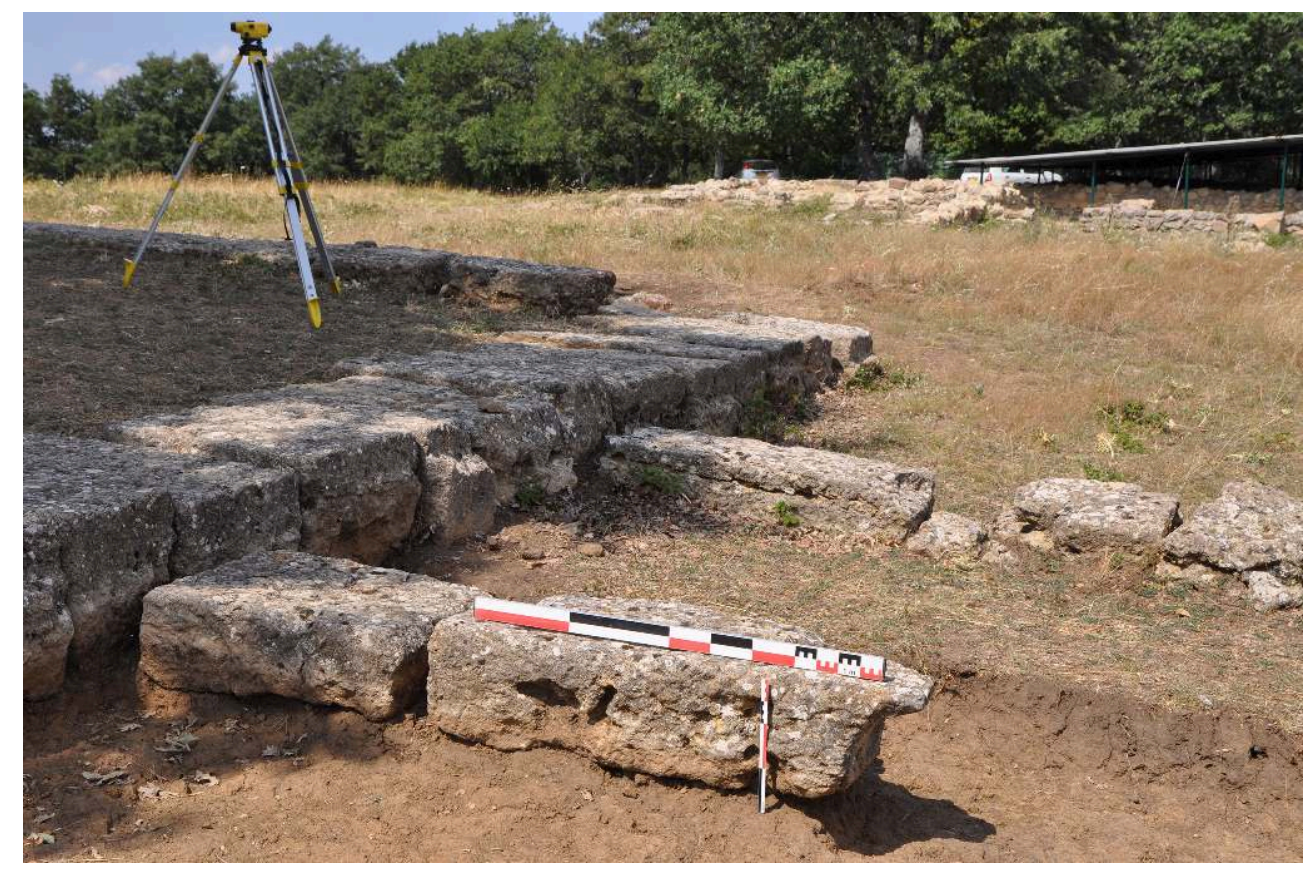

O. de Cazanove.

13 Cette moulure ne pouvait qu'appartenir au podium, et plus précisément à sa corniche supérieure (puisque la moulure inférieure, encore partiellement en place, est un cavet). On peut dire davantage : un bloc est normalement mouluré sur son côté long. C'est d'ailleurs le cas des cavets du temple de l'acropole, sauf aux angles du podium. Les blocs d'angle antérieurs (non conservés) devaient être logiquement moulurés sur deux côtés adjacents, un long et un court. Aux angles postérieurs du podium, par contre, les cavets inférieurs (tous les deux in situ) se trouvent uniquement sur un petit côté des blocs, car le mur du fond ne comportait pas de modénature, sans doute parce qu'il n'était pas en vue. Le bloc en remploi dans le mur d'échiffre présente la même disposition (doucine sur un seul côté court, tandis que les trois autres faces sont verticales). Par conséquent, il devait nécessairement appartenir à la corniche supérieure de l'un des angles supérieurs du podium (nord ou ouest).

Il peut sembler étrange, à première vue, qu'on ait remployé dans l'escalier d'accès au sommet du podium l'un des blocs qui formait justement ce plan supérieur. Quand l'escalier a été construit, le podium n'était évidemment pas en ruine, et donc ses blocs n'étaient pas disponibles pour d'éventuels remplois. Seule solution possible : un bloc qui s'est cassé avant d'être mis en œuvre (ou durant cette opération) et, pour cette raison, écarté et remplacé par un autre. Or, la moulure d'angle amincit le bloc à son extrémité, le rendant plus fragile. De fait, une partie de celle-ci est aujourd'hui brisée. Il ne restait plus, une fois le bloc endommagé, qu'à le remployer utilement à un endroit où ce défaut n'allait être que peu ou pas du tout visible.

On peut, à ce point, proposer une restitution graphique du podium (fig. 9). 
Fig. 9 - Restitution du podium.

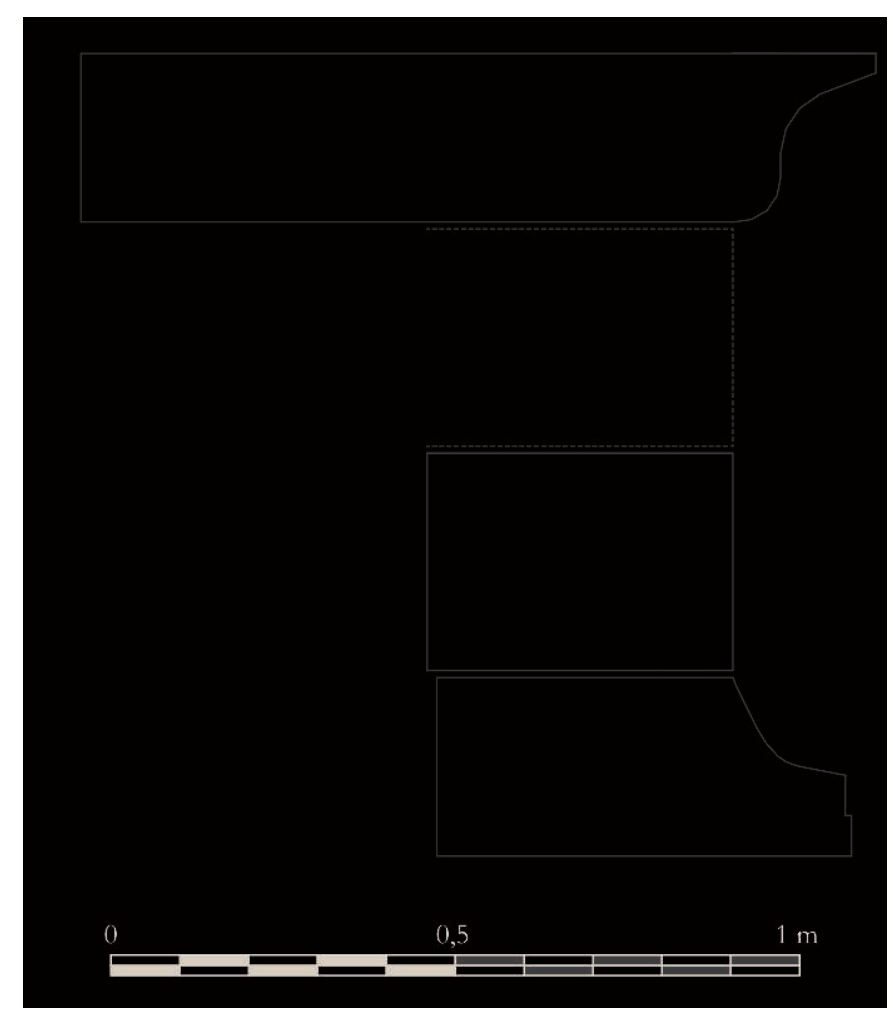

T. Terrasse.

La seule incertitude porte sur la partie médiane de l'élévation du podium, entre les deux moulures. Un seul bloc lui appartenant est conservé in situ. Pour des raisons de proportions, il faut en restituer un deuxième (voire un troisième). On obtiendrait ainsi un podium haut d'env. 1,20 m (ou d'1,50 m). D'ailleurs, les murs d'échiffre de l'escalier sont longs en moyenne de 2,10 m, une longueur suffisante pour y replacer 7 marches avec un giron de $30 \mathrm{~cm}$ (1 pied romain). Si les contremarches étaient hautes de $17 \mathrm{~cm}-$ ce qui représente une valeur moyenne - on obtient un podium haut au total d'1,19 m ou plutôt (le sommet du podium formant une huitième marche, palière) 1,36 m. Nous avons obtenu précédemment, dans l'hypothèse la plus probable, 1,20 $\mathrm{m}$. Si l'on rajoute un stylobate sur lequel les colonnes auraient été placées (et non directement sur le plan correspondant au lit d'attente des corniches), on augmente la hauteur totale du podium jusqu'à atteindre env. 1,35 m.

Le podium ainsi restitué demeure cependant un peu plus bas que ceux du Samnium : $1,47 \mathrm{~m}$ à San Giovanni in Galdo, 1,51 m à Fontecchio, 1,65 m pour le temple $\mathrm{A}$ de Pietrabbondante, 1,83 m à Vastogirardi, sans parler des exemples bien plus imposants de Schiavi (grand temple) et de Pietrabbondante (temple B). Pour ce qui est de la datation du temple d'après les modénatures, le parallèle que j'avais suggéré autrefois avec le temple de Jupiter à Pompéi ${ }^{4}$, de la seconde moitié du II ${ }^{\mathrm{e}}$ s. av.J.-C., devient moins pertinent, parce qu'à Pompéi les moulures supérieure et inférieure sont toutes deux des cavets (droit et renversé). Quoi qu'il en soit, l'emploi de la doucine est certainement l'indice d'une chronologie relativement tardive ${ }^{5}$ et pourrait éventuellement induire à abaisser la chronologie que j'avais proposée ${ }^{6}$.

Le mur 5210 qu'on a rencontré plus à l'ouest, dans une tranchée perpendiculaire au mur du fond du podium, devrait appartenir lui aussi à la phase tardo-républicaine. Il 
correspond sans doute à l'un des murs parallèles visibles sur la prospection géophysique (fig. 4). Sa technique de construction (il est lié au mortier) est différente de celle des autres murs, ainsi que son orientation. Il faisait sans doute partie de l'enclos du temple, en l'isolant de la route qui passait plus à l'ouest (cette dernière joignait la rampe d'accès dallée de l'acropole à la porte fouillée par la Surintendance archéologique en 1996). En 2014, on enlèvera l'épaisse démolition qui s'appuyait au mur 5210. La prochaine campagne fournira donc de nouvelles données sur la phase du temple et surtout sur la phase antérieure.

Fig. 10 - La fouille vue du nord, à la fin de la campagne 2013.

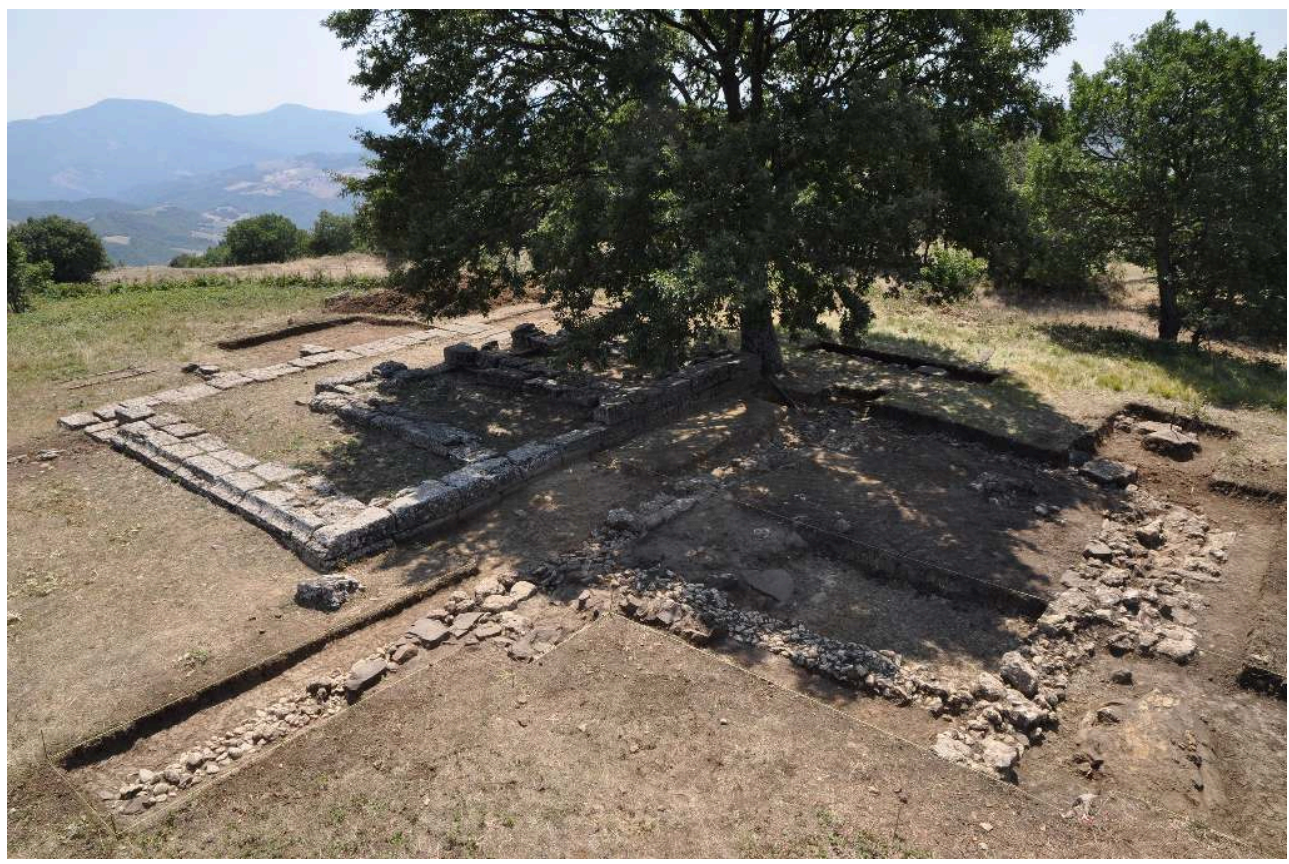

O. de Cazanove.

\section{La prospection géophysique (électrique) dans la parcelle 14 (Olivier de Cazanove et Sylvia Estienne)}

19 Une prospection géophysique (électrique - méthode ARP) a été effectuée sur le site de Civita di Tricarico en octobre 2013, sur la parcelle 14 (privée). La superficie couverte est de 5,7 hectares. Les données ont été acquises sur le terrain par G. Bitella, pour le compte de la société Geocarta qui a élaboré la carte de résistivité à trois profondeurs différentes ( $\mathrm{v}-1, \mathrm{v}-2, \mathrm{v}-3)$. La même société avait déjà réalisé deux prospections sur le plateau de Civita. La première (en 2007-2008) avait porté sur 4,3 hectares dans la « cité basse $»^{7}$. La seconde (en 2010) a imagé 1,75 ha sur l'acropole. Avec la troisième, la superficie couverte atteint en tout 11,75 ha (fig. 11). Or, la superficie du site intramuros peut être estimée à 47 ha, dont 31 env. constructibles. Par conséquent, on peut considérer qu'est désormais connue, par la fouille ou la prospection, un peu moins de la moitié « utile » du site. 
Fig. 11 - Les prospections géophysiques (électriques) réalisées de 2007 à 2013 sur le site de Civita di Tricarico.

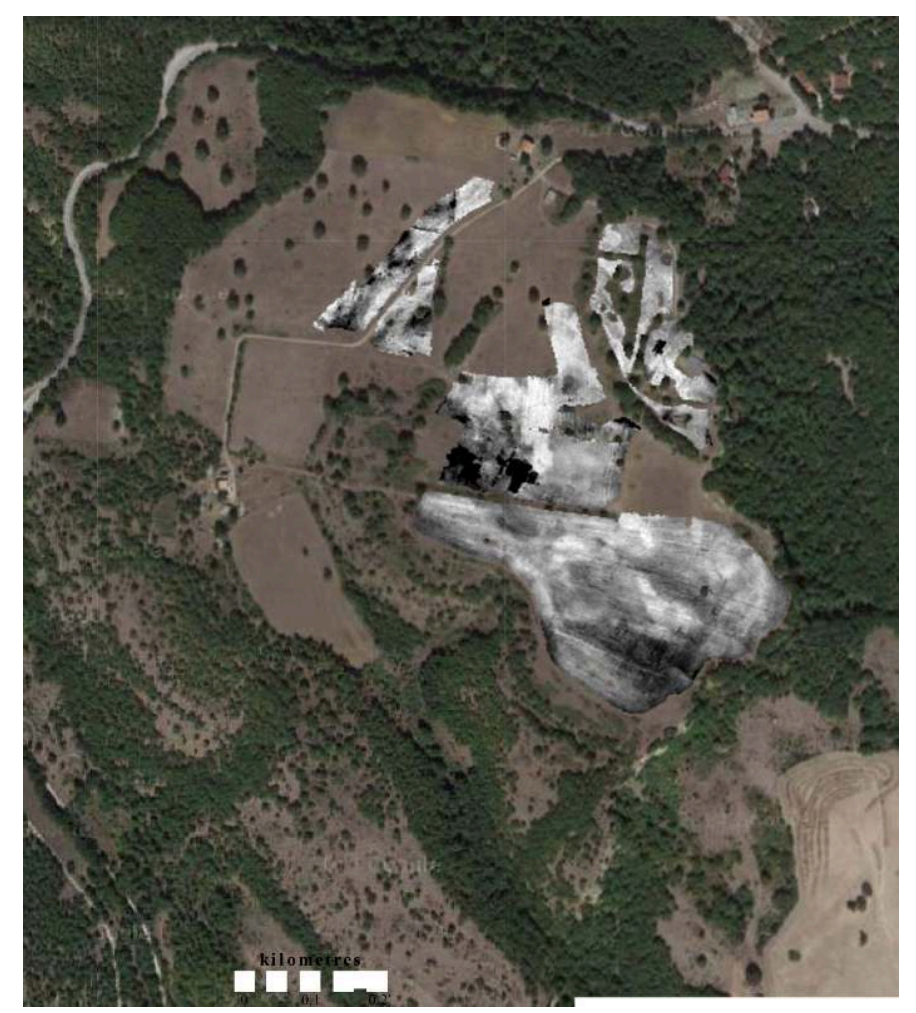

Geocarta.

Le recours à la prospection géophysique s'imposait d'autant plus que les photographies aériennes, obliques ou zénithales, n'avaient jamais révélé de structures sous-jacentes sur le plateau. Du moins jusqu'à celles prises en 2007-2008 par B. Callahan, qu'il faut ici chaleureusement remercier pour les avoir aimablement communiquées. Redressées et filtrées, elles viennent d'être publiées ${ }^{8}$ (on en publie ici une autre image, traitée comme les précédentes par J.Vidal que nous remercions également: fig. 12). On distingue plusieurs édifices rectangulaires, dont les modules et les plans sont assez similaires. L'impression - peut-être trompeuse - est celle de "maisons à pastas élémentaires ", mais la pastas devrait être plutôt au sud qu'au sud-est. D'autres structures, plus petites et isolées, se trouvent davantage au nord-est. 
Fig. 12 - Parcelle 14, photographie aérienne (de Brad Callahan) redressée. Filtrage avec bande de couleur réduite.

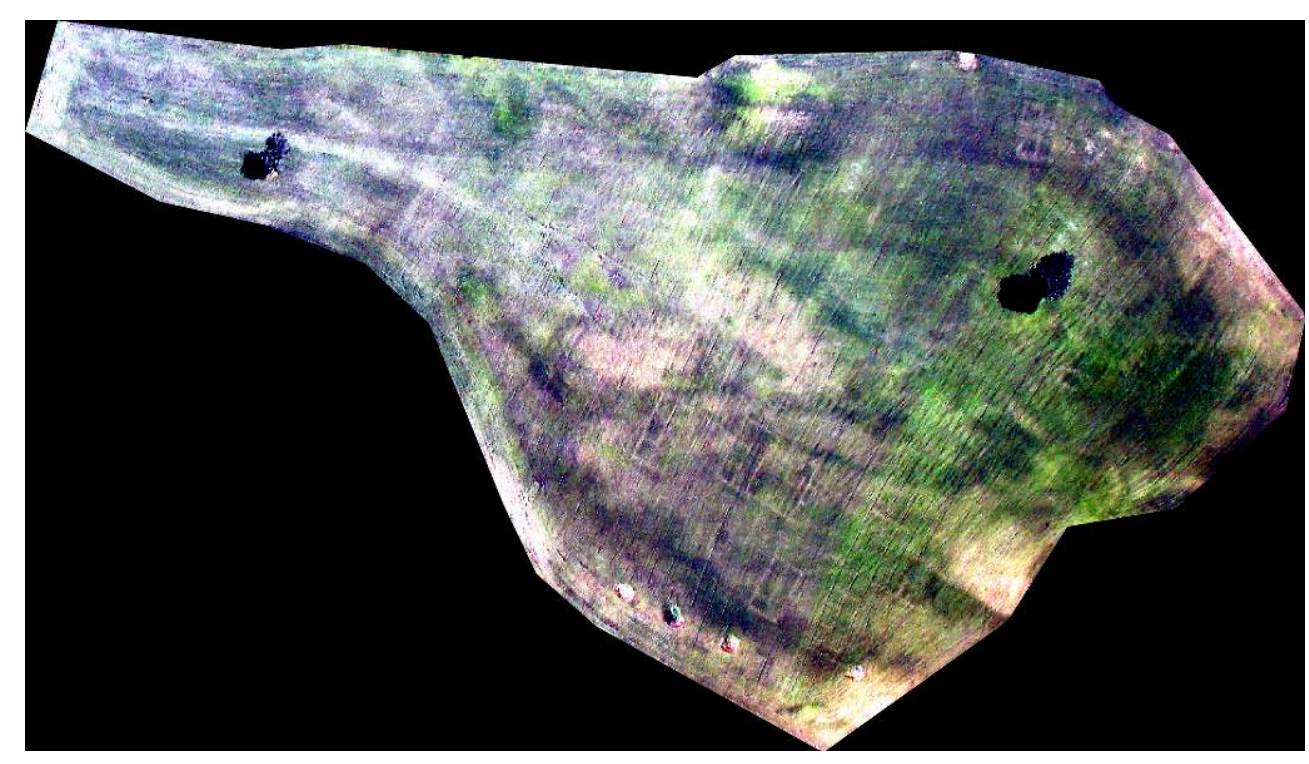

J. Vidal, UMR 6298 ArTeHiS.

21 Ces nouvelles données appelaient une confirmation. Non seulement pour vérifier l'existence effective de ces maisons - et en découvrir éventuellement d'autres - en utilisant un autre moyen d'enquête extensive (alors qu'il était exclu a priori de recourir à des sondages de fouille, car il s'agit de terrains privés); mais également pour améliorer le redressement des photos obliques et les géoréférencer précisément; et enfin pour valider mutuellement les deux méthodes. En effet, si elles donnaient le même résultat, "l'évidence positive » (les traces de murs) devrait être définitivement admise; là où, au contraire on rencontrerait, quelle que soit la technique employée, une " évidence négative » (une absence de traces), il faudrait selon toute probabilité en déduire un vide réel et non une lacune documentaire. Des trois cartes de résistivité, qui sont substantiellement identiques malgré de légères variations, celle de la voie 3 , la plus profonde, est aussi la plus claire (fig. 13). On peut y discerner les édifices visibles sur la photographie aérienne et probablement quelques autres. 
Fig. 13 - Parcelle 14, carte de résistivité, voie 3 avec l'indication des principaux édifices reconnus.

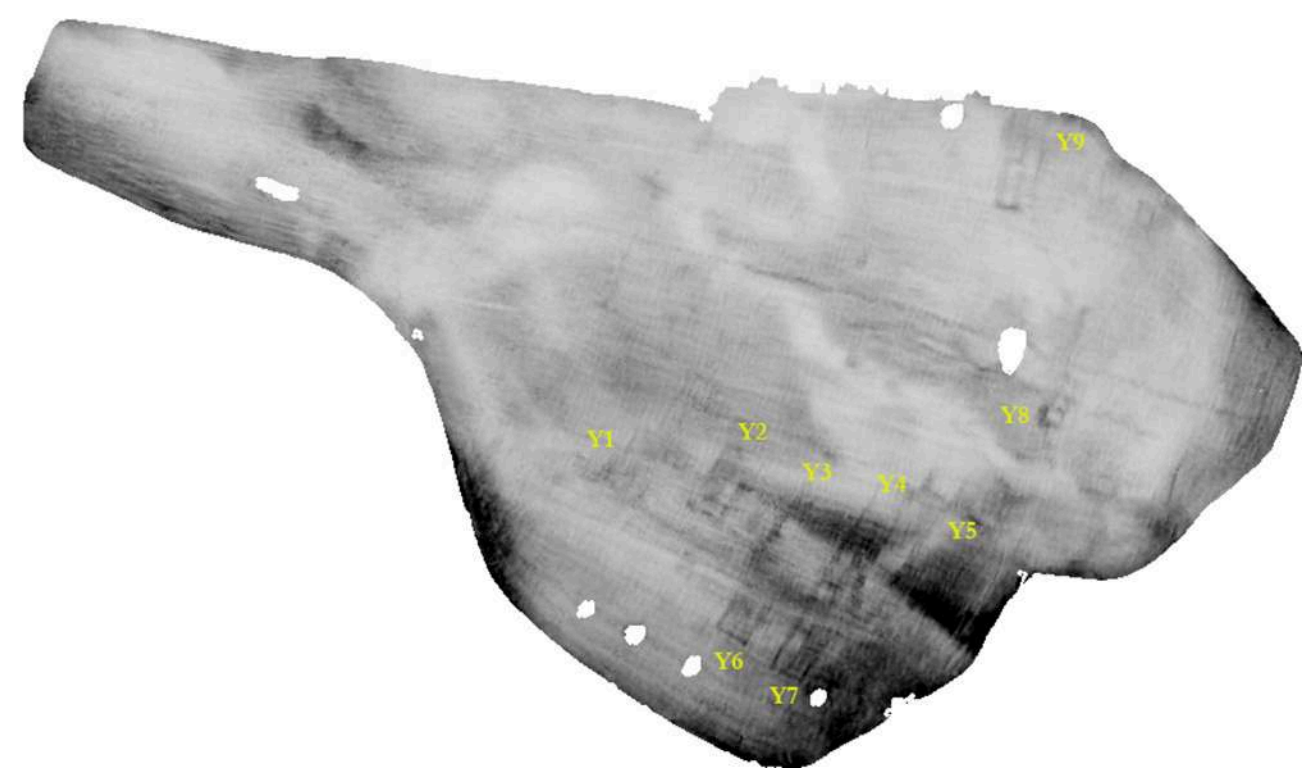

Geocarta et O. de Cazanove.

La combinaison des données de la photographie aérienne et de l'enquête géophysique révèle donc, à Civita di Tricarico, l'existence, jusque-là insoupçonnée, d'un " quartier » entièrement nouveau. Il est préférable de parler ici de "quartier" plutôt que de « noyau ». Le modèle ne semble pas être en l'occurrence l'habitat " polynucléaire » ou "polycentrique ", avec plusieurs noyaux indépendants les uns des autres, chacun d'eux gravitant autour d'un pôle qui serait une demeure aristocratique (selon un modèle d'interprétation encore couramment employé mais qui ne nous semble plus correspondre, ni à l'état actuel de la recherche, ni à la réalité de faits). D'abord, la logique planimétrique n'est pas centripète, les maisons ne se disposent pas plus ou moins en cercle autour d'un édifice plus important. Elles sont au contraire alignées (sur au moins deux files), et font partie d'une trame qui est plus orthogonale et régulière que partout ailleurs à Civita di Tricarico. En deuxième lieu, leur orientation est la même que celle des autres zones construites du plateau et leur typologie semble identique (quoiqu'elles s'ouvrent vers le sud-est). En d'autres termes, même s'ils sont séparés par 100 à 150 mètres, les différents secteurs de l'agglomération appartiennent à la même trame, au même système d'ensemble. C'est pourquoi il est licite de parler de « quartiers».

Mais s'agit-il vraiment d'un quartier de l'agglomération lucanienne? On pourrait à la rigueur en douter, la prospection géophysique n'étant par définition ni datée ni phasée. Ce groupe de constructions isolées à l'extrémité sud du terrain cultivable, en position dominante, au-dessus d'une source qui jaillit au pied de la falaise, ne pourrait-il appartenir à une période postérieure à la disparition du site de hauteur fortifié ? Par exemple une ferme romaine, des édifices d'époque byzantine, voire une propriété du clergé de Tricarico? Cette possibilité, qu'on ne mentionne ici que pour les évoquer toutes, doit vraisemblablement être exclue. Le plan, tel qu'il apparaît désormais, montre des maisons, des îlots, un quartier de type "urbain " qui répond dans ses grandes lignes aux principes de l'urbanisme régulier antique. On a affaire à un ensemble de maisons disposées de manière ordonnée, et non à un complexe isolé. Le 
matériel de surface trouvé en prospection depuis 25 ans dans la parcelle 14 est exclusivement hellénistique. Il n'est ni romain (impérial) ni plus tardif.

Le quartier de la parcelle 14 est avant tout formé de deux rangées de maisons (plus peut-être une rangée de constructions plus petites, au nord), pour une largeur totale d'env. $55 \mathrm{~m}$ et une longueur d'au moins $100 \mathrm{~m}$, donc une superficie minimale de $5500 \mathrm{~m}^{2}$, mais sans doute davantage. La largeur est approximativement la même que celle de la bande construite le long de la "rue méridionale " ${ }^{9}$. Mais le quartier de la « rue méridionale » est nettement plus allongé : on le suit sur env. $250 \mathrm{~m}$ de longueur.

Une rue semble séparer les deux rangées de maisons. Toutefois, la trace claire que l'on note sur les images v-1 - v-2 - v-3 (mais pas sur la photographie aérienne) présente un tracé sinueux qui contraste avec le plan rigidement géométrique des maisons. On pourrait alors penser à une trace postérieure, peut-être à un parcours qui reprendrait, mais de manière approximative et déformée, le tracé de la voie contemporaine des constructions. Ce pourrait être aussi le cas d'un autre parcours sinueux, visible cette fois sur la photographie aérienne (fig. 13), dans la partie nord du champ.

La majeure partie des édifices semble présenter un plan similaire à celui de la « maison des moules » (ou de la maison $\mathrm{M})^{10}$, mais avec l'ouverture au sud-est. Les dimensions et les modules sont les mêmes : on comparera les $16,80 \times 11,25 / 11,85 \mathrm{~m}$ de la maison des moules (pour une superficie de $199 \mathrm{~m}^{2}$ ), ou encore les 23,04 x 11,07 m de la maison M (sup. $255 \mathrm{~m}^{2}$ ), avec les $23 \times 13,5 \mathrm{~m}$ restituables $\left(310 \mathrm{~m}^{2}\right)$ de la maison Y1. On notera en outre que, tandis que cette maison est l'une de celles qui se voient le mieux sur la photographie aérienne, avec une grande salle (env. $9 \times 7 \mathrm{~m}$ ) au centre du côté nordouest, flanquée par deux pièces carrées symétriques ( $7 \mathrm{~m}$ de côté), le même édifice se laisse à peine deviner sur les cartes de résistivité. Sur celles-ci, on entrevoit mieux la possible trace d'un autre édifice (?) plus au nord-est qui par contre est invisible sur la photographie aérienne.

Pour la maison Y6 on peut restituer les dimensions suivantes : $18 \times 11 \mathrm{~m}\left(198 \mathrm{~m}^{2}\right)$. La maison Y7 est similaire: $17 \times 11 \mathrm{~m}\left(187 \mathrm{~m}^{2}\right)$. On note, encore une fois, la même tripartition des pièces nord-ouest. Un autre fait digne de mention est l'intervalle qui sépare Y5 et Y6: env. $11 \mathrm{~m}$. Comme on l'a observé ailleurs ${ }^{11}$, les maisons sont précédées d'une cour extérieure de même largeur. Cette cour peut ensuite être envahie de constructions, complètement ou en partie. Cela semble avoir été le cas de la maison Y2. $\mathrm{Au}$ nord-ouest, on retrouve l'habituelle "maison à pastas élémentaire " avec trois pièces qui s'ouvrent sur la pastas $\left(19 \times 12 \mathrm{~m}=228 \mathrm{~m}^{2}\right)$. Au sud-est, on a une extension un peu plus grande ( $19 \times 14 \mathrm{~m})$, avec un plan plus irrégulier et avec un mur périmétral sud qui ne semble pas exactement aligné sur le côté court de la maison Y2. Au total, la maison Y2, extension comprise, fait $26 \times 20 \mathrm{~m}$.

Un étroit ambitus, bien visible à la fois sur la photographie aérienne et sur la carte géophysique, sépare la maison Y2 de la Y3 dont on voit peu de chose, à l'exception du mur nord-ouest, dont partent des diviseurs (peut-être trois). La maison Y3 semble s'aligner exactement sur Y6. Il en va de même pour Y4, alignée sur Y7. La rangée nord semble compter une cinquième maison, $\mathrm{Y} 5$, proche de la falaise.

Le reste de la parcelle 14 paraît dépourvu de constructions, à part deux édifices isolés dans la partie est du champ : Y8, apparemment monocellulaire, et Y9, étroit et allongé dans le sens nord-sud. 
Il faut revenir, en conclusion, sur la régularité de la trame, assurément supérieure à celle qu'on observe dans les autres secteurs habités de Civita. Ce fait, en même temps que les bonnes conditions dont a bénéficié la prospection, explique la lisibilité exceptionnelle d'un plan qui peut être décrit avec précision. En définitive, la combinaison des méthodes d'enquête permet de restituer un quartier entier sans devoir recourir à la fouille.

\section{Prospections sur les territoires de Tricarico, San Chirico Nuovo et Tolve (Stéphane Bourdin)}

\section{Prospections autour du site de Civita di Tricarico}

31 Dans un premier temps, l'enquête a porté sur l'enceinte externe, pour en localiser avec précision les portions les mieux conservées. Sur le versant oriental de l'acropole, en partant de la porte, des portions, entrecoupées de secteurs écroulés, sont encore visibles. Dans le sous-bois en dessous (zone AA), on a découvert une grande quantité de matériel (tuiles, céramique etc.), provenant des secteurs effondrés de l'acropole, notamment des environs de la domus, dont l'angle oriental est très proche de la falaise. On voit encore sur le bord de celle-ci un gros fragment de tuile à opaion, qui provient d'un des édifices de l'acropole. Aucune fortification n'est nécessaire sur tout le secteur oriental, en raison de la présence de la falaise, à l'exception d'un secteur où la hauteur de la paroi diminue. À cet endroit, on ne voit pourtant qu'une portion de murs en petits moellons de calcaire, probablement remonté. De gros blocs écroulés dans la partie orientale, près de la masseria Rocchetta, indiquent que le plateau a subi, jusqu'à une époque récente, des effondrements importants et que la surface originelle devait être supérieure à la superficie actuelle (47 ha). 
Fig. 14 - La falaise sur le versant SE du plateau.

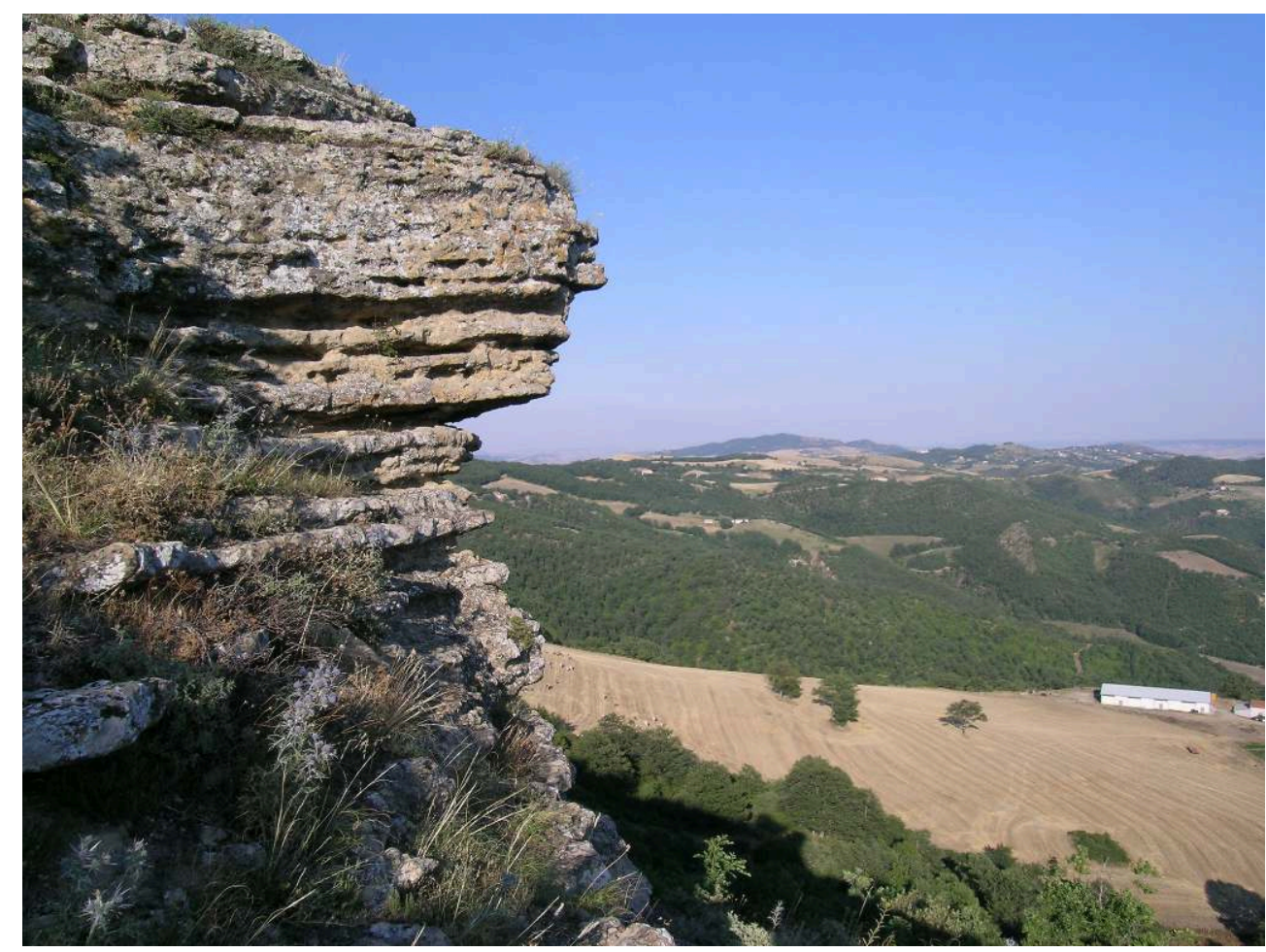

S. Bourdin.

32 Au niveau du vallon dit "Carnaleta », emprunté par un sentier, l'accès au plateau devient plus aisé. Sur le versant occidental, près de la masseria Perone, nous avons pu localiser certaines portions de l'enceinte externe et de l'enceinte intermédiaire, qui n'avaient pas encore été repérées avec précision. Sur le versant septentrional en revanche, la fortification a été en grande partie démontée et on ne voit aucune trace d'enceinte, malgré la faible pente par endroits; seule la présence d'un léger décrochement entre le bord du champ et le versant permet de supposer la présence de l'enceinte à ce niveau.

Trois sources ont été repérées au pied de la falaise : la source du Sambuco, qui est en partie captée pour alimenter la masseria Rocchetta, une autre qui sourd au fond d'une des grottes, et une dernière qui alimente un abreuvoir au sud-ouest. Plusieurs grottes ou abris sous roche sont présents le long de la falaise. Le premier abri se trouve à proximité de la source du Sambuco et il est fermé par un muret moderne. Au sud se trouvent trois grottes : dans la première coule la source, dont les eaux sont recueillies par une petite canalisation jusqu'à un mur qui barre l'entrée et qui servait peut-être à soutenir un bassin. La $2^{\mathrm{e}}$ grotte est profonde de $39,82 \mathrm{~m}$, pour une largeur maximale de $9,73 \mathrm{~m}$ et une hauteur maximale de $2,60 \mathrm{~m}$. Elle est occupée par un édifice troglodytique, constitué d'une petite pièce, ouverte vers l'extérieur $(3,88 \times 4,26 \mathrm{~m})$, flanquée d'une longue étable $(3,88 \times 17,99 \mathrm{~m})$. Sur le côté droit, une petite rigole permet l'écoulement de l'eau hors de la grotte. La $3^{\mathrm{e}}$ grotte, qui porte encore le numero civico 4, mesure 14,71 $\mathrm{m}$ de longueur pour une hauteur maximale de 3,44 m. L'entrée est fermée par un mur moderne et une banquette a été creusée sur la paroi de droite. De nombreuses légendes courent sur ces grottes, dont l'une qui prétend qu'elles communiquent avec la grotte du brigand "Carluccio », située au nord du plateau, à 
$958 \mathrm{~m}$ de distance à vol d'oiseau. Cette dernière correspond en réalité à un puits karstique, profond de 4 à $5 \mathrm{~m}$.

Fig. 15 - Grotte sur le versant SE.

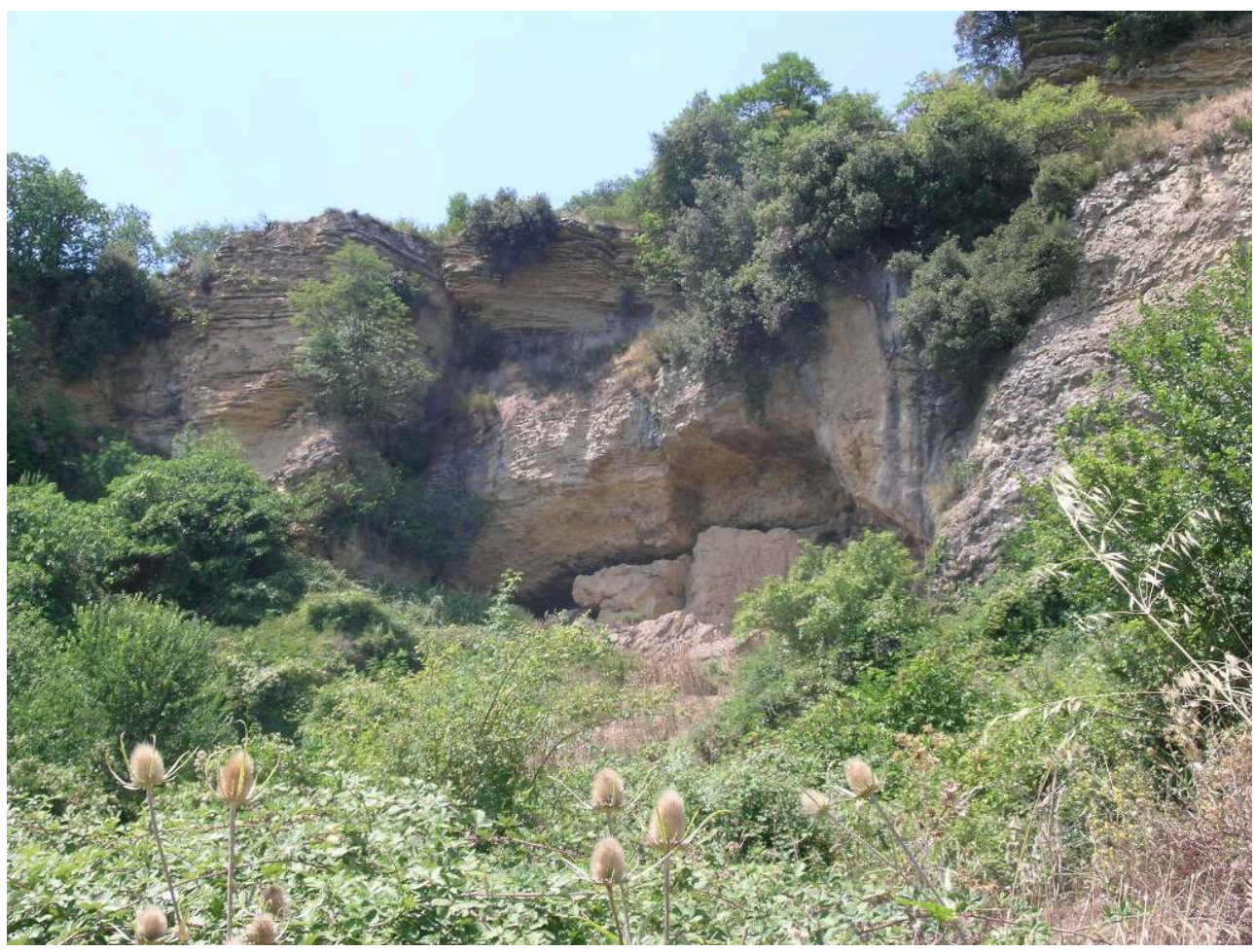

S. Bourdin 
Fig. 16 - Grotte sur le versant SE.

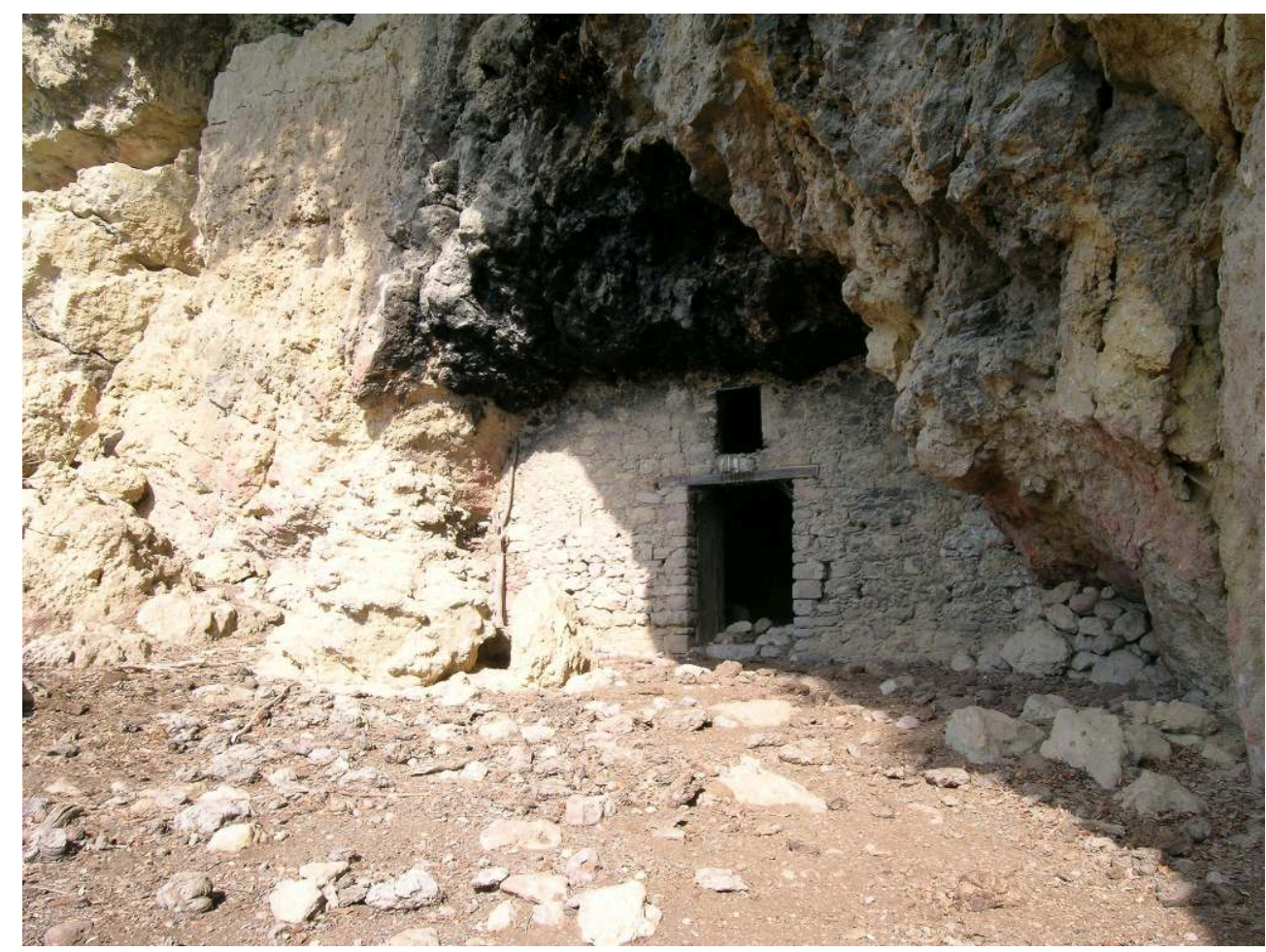

S. Bourdin.

Le lieu de provenance de l'inscription Vetter $183=\mathrm{ST}$ Lu 13, que l'on attribuait à la masseria Rocchetta, a été localisé avec précision d'après les témoignages des habitants. Cette inscription aurait en revanche été découverte lors de la construction d'un cabanon adjacent à l'autre ferme, propriété de la famille Di Dio, plus au sud. Durant la construction d'un hangar, on a récemment mis au jour des portions de murs et toute la zone a livré des blocs de calcaire taillés, de la céramique et des pesons. On peut donc supposer la présence d'un petit sacellum, à faible distance de la pointe méridionale de l'enceinte de Civita et à $407 \mathrm{~m}$ du débouché du vallon de Carnaleta, où s'ouvrait peutêtre une poterne.

35 Si l'enceinte a en grande partie disparu sur le versant occidental, des blocs équarris $(78 \times 29,5 \times 34 \mathrm{~cm})$ ont été récupérés et réutilisés pour soutenir la route SS 7. D'autres blocs de même module se rencontrent, au milieu de fragments de tuiles, dans le bois de Pisciolo, au nord de la route. 
Fig. 17 - Blocs de l'enceinte en réemploi sous la route SS 7.

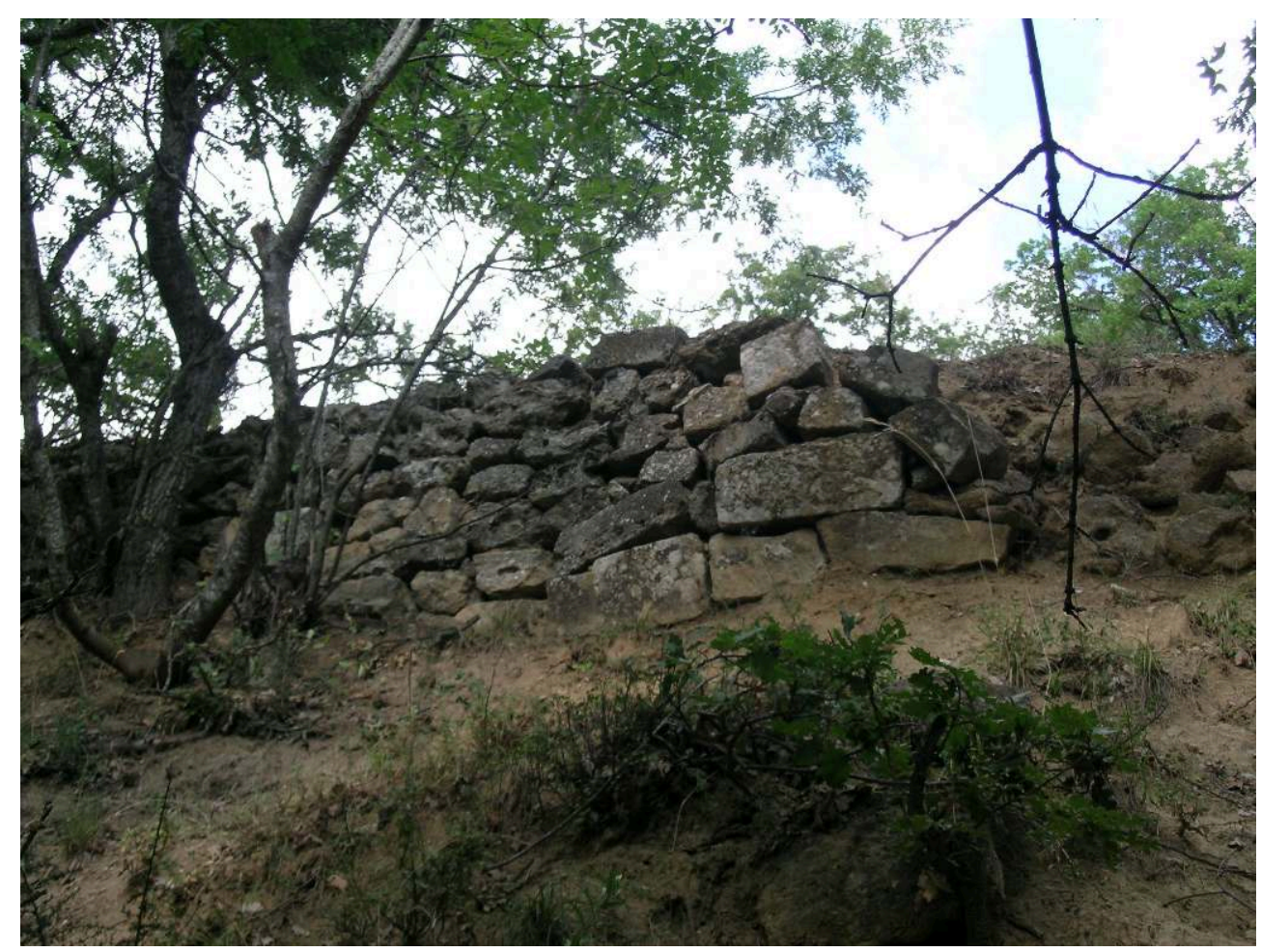

S. Bourdin.

Tout le secteur du Monte La Pila (932 $\mathrm{m}$ d'altitude), qui domine le col de Tre Cancelli où sont localisées les nécropoles, a été exploré, mais la construction récente de maisons a irrémédiablement compromis la situation antérieure.

Enfin, le massif du Monte Cupolicchio (1097 m) a été exploré, depuis les sommets de Crocetta (1027 et $1035 \mathrm{~m}$ ) jusqu'au Monte Vitale $(1064 \mathrm{~m})$ et au lieu-dit Campagnasco $(1068 \mathrm{~m})$. Aucune trace d'occupation antique, à l'exception d'un probable menhir, n'a été mise en évidence. Les seules traces d'anthropisation repérées sont des petites vasques creusées dans la roche pour récupérer l'eau de pluie, ainsi qu'un abreuvoir rupestre, formé de deux vasques, l'une rectangulaire $(2,10 \times 1,64 \mathrm{~m})$ et l'autre grossièrement triangulaire $(1,20 \mathrm{~m}$ de longueur maximale pour sa «bissectrice »), réunies par un conduit d'écoulement. Ces structures témoignent de la fréquentation pastorale du secteur, désormais recolonisé par la chênaie. 
Fig. 18 - Abreuvoir rupestre du Monte Cupolicchio.

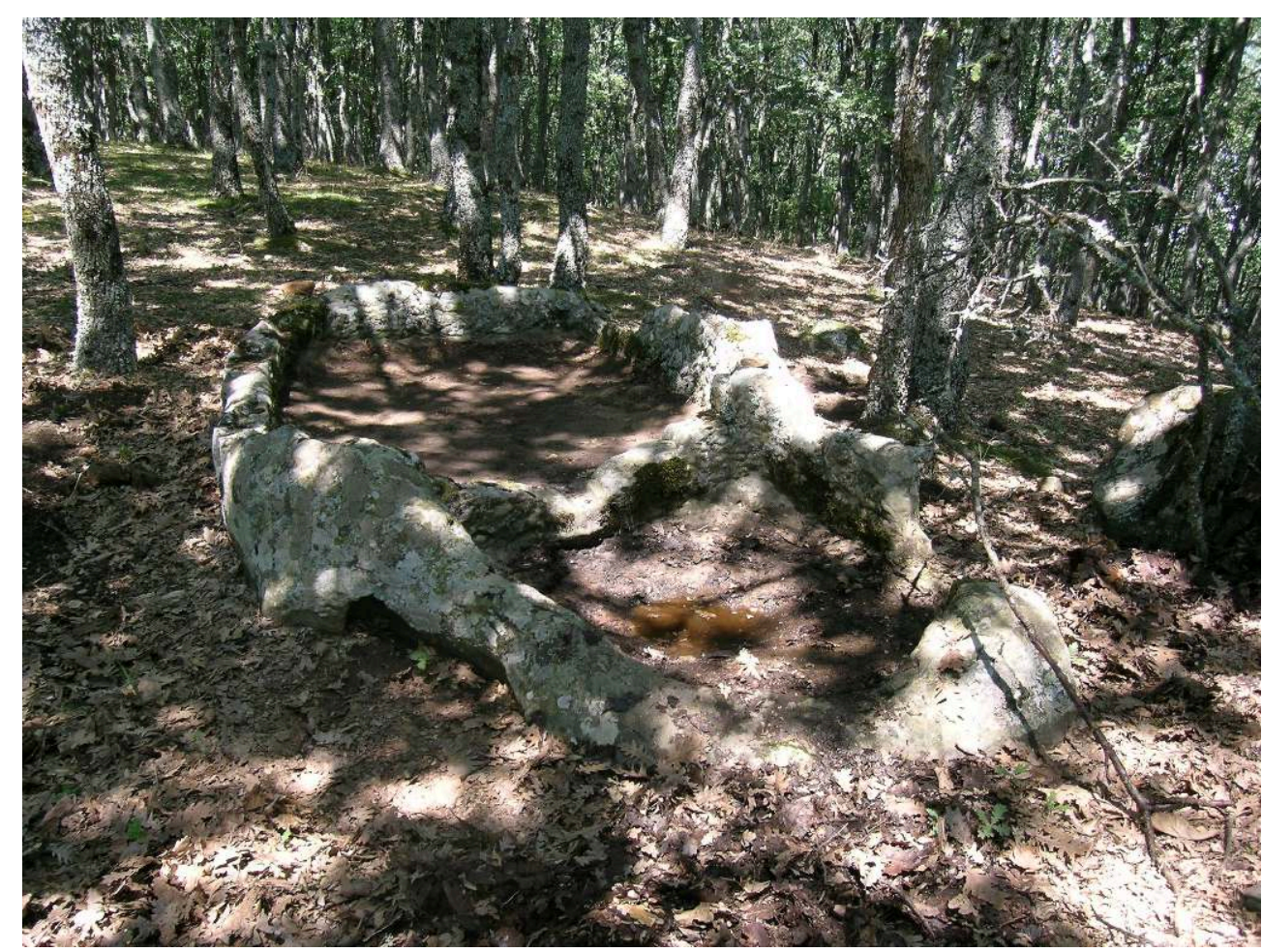

S. BOURDIN.

\section{Prospections à Serra del Cedro (Tricarico)}

Le site de Serra del Cedro a été partiellement exploré par la Surintendance, avec la fouille d'une partie de la nécropole et le contrôle des travaux (en 1986) en vue de la réalisation d'un gazoduc. À cette occasion, la présence de maisons, avec des murs sur solin de pierres sèches a pu être documentée. En complément, nous avons tenté de vérifier la présence d'une éventuelle fortification. Si le matériel présent en surface (tegulae, imbrices, pesons, céramique etc.) atteste l'occupation dense du site, aucune trace claire d'enceinte n'a été mise en évidence. Sur le versant ouest, on note la présence de blocs équarris, mais les épaulements le long desquels ils s'alignent correspondent en fait aux sillons des tranchées de reboisement. Au nord-est, on a repéré des tas d'épierrement et des terrasses agricoles, organisées en lignes concentriques, qui reprennent peut-être partiellement le tracé d'une enceinte antique.

\section{Prospections à San Chirico Nuovo}

39 La colline de Cugno Notaro $(782 \mathrm{~m})$ / La Serra $(797 \mathrm{~m})$, située à la sortie du village moderne, a fait l'objet de fouilles qui ont révélé la présence d'un édifice quadrangulaire, dont la pièce centrale était occupée par des pithoi, ainsi que celle de tombes du Ves. av. J.-C. ${ }^{12}$ Toute la zone est actuellement occupée par des champs labourés et par une maison, tandis que les deux sommets ont vu apparaître des antennes téléphoniques et un anémomètre pour le premier et une maison pour le second. Toute la colline est en outre traversée par la tranchée d'un gazoduc, le long de laquelle on peut voir, en surface, un abondant matériel céramique et métallique (pointe 
de lance de fer), qui fait écho à la présence de tombes signalées par le passé. Sur le versant nord, connu comme Serra del Barone, une piste descend vers la Fonte del Barone et Tolve. Cet itinéraire se poursuivait probablement sur le versant sud, jusqu'au niveau du village actuel de San Chirico et suivait peut-être un parcours antique. Cette portion méridionale a été effacée par la constitution d'une grande parcelle, aujourd'hui cultivée.

Des prospections ont été effectuées sur la colline, en distinguant les zones AH (sommet secondaire, autour des antennes), AI (sommet principal, autour de l'anémomètre), AJ (zone basse entre les deux sommets), AK (champ au nord de la route, à Serra del Barone), AL (moitié orientale du grand champ). La zone AK correspond à la moitié orientale du grand champ du versant sud, soit les parcelles cadastrales 150-152, 164-165, et couvre 2,8 ha; la zone AH-AI-AL correspond aux parcelles 471 et 481, partiellement étudiées sur 3,84 ha. Les prospections ont révélé une occupation dense, avec un matériel concentré en diverses poches, qui font penser à la présence de groupes d'habitation, sur les deux versants et sur les sommets. L'occupation du versant méridional semble arriver jusqu'à la route moderne et on trouve encore du matériel (fragment de tuiles, pesons, céramique commune etc.) plus au sud dans les environs de l'abreuvoir dit « la Pila » (zones AF et AG).

Fig. 19 - San Chirico Nuovo, Cugno Notaro : zones de prospection pédestre.

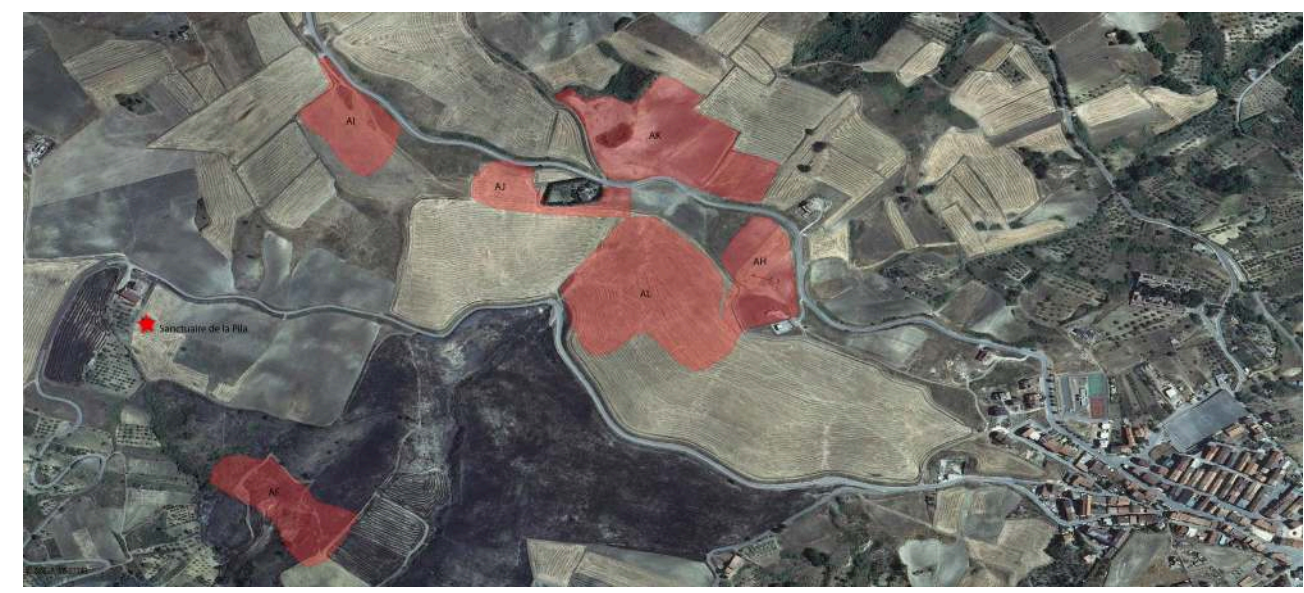

S. Bourdin, d'après image Google Earth.

41 Dans tout ce secteur, et particulièrement dans les zones AG, AI, AJ et AK, on voit une grande quantité de matériel d'époque archaïque (plutôt au sommet) et hellénistique, avec de la céramique à bandes peintes (matt painted), achrome, à vernis noir, de cuisine etc.

42 Ce matériel fait penser à la présence d'un habitat étendu sur une dizaine d'hectares, mais d'autres prospections seront nécessaires pour identifier clairement les limites méridionale et surtout occidentale de l'occupation. Il semble pour l'instant évident que l'occupation se poursuit sur le versant sud jusqu'à la route moderne, presque jusqu'au contact avec le sanctuaire fouillé dans les années 1990 par la Surintendance au lieu-dit Pila ${ }^{13}$, et actuellement recouvert par un cabanon et un potager.

La colline de Serra Lentine, à cheval entre les communes de Tricarico et San Chirico Nuovo, culmine à $854 \mathrm{~m}$ d'altitude et domine la vallée du torrent Bilioso. Les prospections conduites dans les champs labourés sur le versant méridional et sur la 
ligne de crête n'ont révélé qu'un peu de matériel épars (tuiles) et résiduel. Dans le champ labouré immédiatement au NO du sommet, en revanche, on peut voir une quantité notable de vestiges (tegulae, imbrices, céramique à argile dépurée et céramique de cuisine). Le matériel semble être concentré dans le bosquet au sommet, autour du point géodésique, en particulier là où un tracteur a creusé un chemin d'accès au champ, traversant la cime dans le sens sud-nord (zone $\mathrm{AE}$ ), où l'on en trouve une grande quantité (céramique à vernis noir, fine etc.). L'ensemble fait penser à la présence d'un petit édifice, couverte de tuiles, situé au sommet de la colline à l'époque hellénistique.

Fig. 20 - Serra Lentine : zones de prospection pédestre.

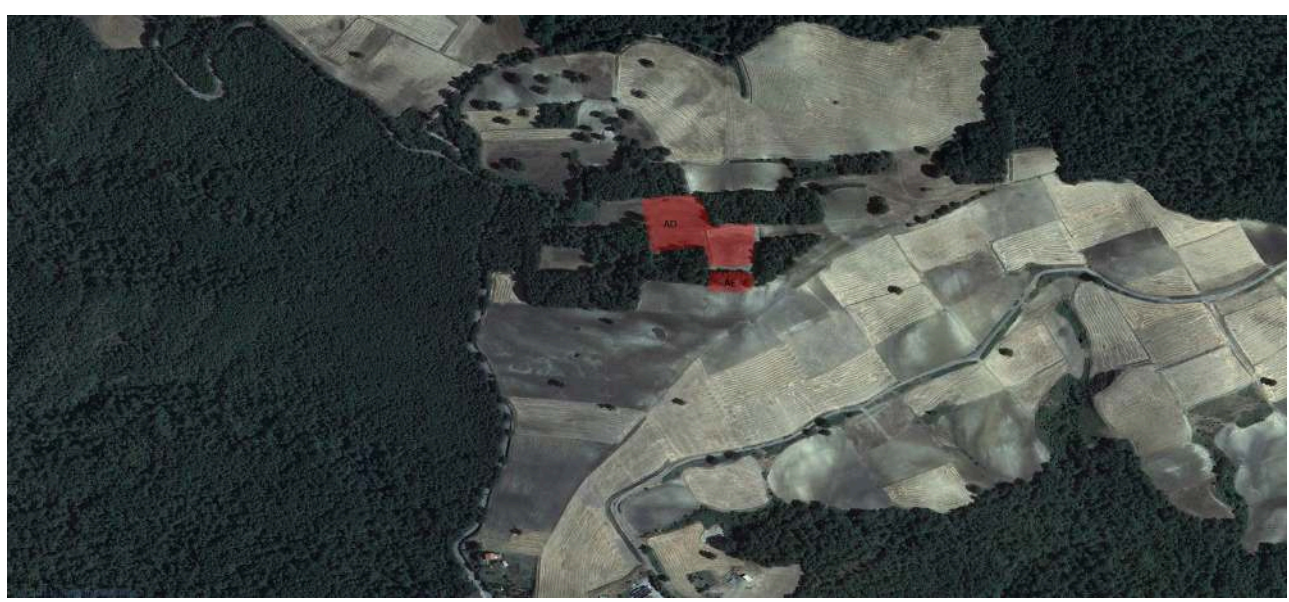

S. Bourdin, d'après image Google Earth.

\section{Prospections à Tolve}

Sur la commune de Tolve, le secteur autour de la masseria Santa Maria (502 m) et de la chapelle de Sant'Antonio Abate (609 m) a été exploré, sans résultat significatif.

\section{BIBLIOGRAPHIE}

Adamesteanu 1974 = D. Adamesteanu, L'attività archeologica in Basilicata, dans Metaponto. Atti XIII ${ }^{\text {mo }}$ convegno di studi sulla Magna Grecia. Taranto 14-19 ottobre 1973, Naples, 1974, p. 441-456.

Bottini - Pica 1984 = A. Bottini, E. Pica, dans SSc, SE, LII, 1984 (1986), p. 483-483.

Canosa 1990 = M. G. Canosa, Tricarico, dans M. Salvatore (éd.), Basilicata. L'espansionismo romano nel sud-est d'Italia : il quadro archeologico, Atti del convegno, Venosa, 23-25 Aprile 1987, Venosa, 1990, p. 111-123. 
Cazanove 2001 = O. de Cazanove, Civita di Tricarico nell'età della romanizzazione, dans E. Lo Cascio, A. Storchi Marino (éd.), Modalità insediative e strutture agrarie nell'Italia meridionale in età romana, Bari, 2001, p. 169-202.

Cazanove 2004 = O. de Cazanove, Un nouveau temple à Civita di Tricarico (Lucanie), dans MEFRA, 116, 1, 2004, p. 249-291.

Cazanove 2008 = O. de Cazanove, Civita di Tricarico. 1. Le quartier de la maison du monolithe et l'enceinte intermédiaire, Rome, 2008 (Collection de l'École française de Rome, 409).

Cazanove 2009 = O. de Cazanove, Edifici di culto lucani a pianta centrale quadrata, dans Siris, 10, 2009, p. 131-141.

Cazanove - Dabas - Sarro 2008 = O. de Cazanove, M. Dabas, L. Sarro, Civita di Tricarico (prov. de Matera), dans MEFRA, 120, 1 [Chronique des activités de l'École française de Rome], 2008, p. 253-256.

Cazanove - Féret - Caravelli 2014 = O. de Cazanove, S. Féret, A. M. Caravelli, Civita di Tricarico II. Habitat et artisanat au centre du plateau, Rome, 2014 (Collection de l'École française de Rome, 483).

Gros 1996 = P. Gros, L'architecture romaine, du début du III ${ }^{e}$ s. av. J.-C. à la fin du Haut-Empire. I. Les monuments publics, Paris, 1996.

Tagliente 1998 = M. Tagliente, Il santuario di San Chirico Nuovo, dans Il Sacro e l'Acqua. Culti indigeni in Basilicata (Mostra Roma, Museo Barracci 23 aprile-18 ottobre 1998), Roma, 1998.

\section{NOTES}

1. Cazanove 2004 et 2009.

2. Adamesteanu 1974, p. 448-449.

3. Canosa 1990, fig. 2.

4. Cazanove 2001, p. 195.

5. Gros 1996, p. 134.

6. Cazanove 2001, p. 189-195, avec une datation dans la deuxième moitié du II ${ }^{\mathrm{e}}$ s. av. J.-C.

7. Cazanove - Dabas - Sarro 2008.

8. Cazanove - Féret - Caravelli 2014, p. 38-41.

9. Cazanove - Féret - Caravelli 2014, p. 34 et suiv.

10. Sur celles-ci, Cazanove - Féret - Caravelli 2014.

11. Cazanove 2008, p. 63-64.

12. Bottini - Pica 1984.

13. Tagliente 1998. 
INDEX

Mots-clés : temple, prospection géophysique, prospection pédestre, San Chirico Nuovo institutions École française de Rome, Université de Paris I Panthéon-Sorbonne, Labex TransferS École normale supérieure, UMR 7041 ArScAn, Soprintendenza per i beni archeologici della Basilicata

Index géographique : Civita di Tricarico, Rossano di Vaglio, Serra del Cedro

\section{AUTEURS}

\section{OLIVIER DE CAZANOVE}

Université de Paris I Panthéon-Sorbonne - cazanove[at]univ-paris1.fr

\section{STÉPHANE BOURDIN}

Directeur des études pour l'Antiquité, École française de Rome - dirant[at]efrome.it 